\title{
Overview of the Investigation and Management of Cystic Neoplasms of the Pancreas
}

\author{
Robert A.J. Spence ${ }^{a}$ Bobby Dasari ${ }^{a}$ Mark Love $^{b}$ Barry Kelly $^{b}$ Mark Taylor ${ }^{a}$ \\ ${ }^{a}$ Hepatobiliary Unit, Mater Hospital, and ${ }^{b}$ Department of Radiology, Royal Victoria Hospital, Belfast, UK
}

\section{Key Words}

Pancreas $\cdot$ Cysts $\cdot$ Neoplasia

\begin{abstract}
Background: Cystic neoplasms of the pancreas contribute to $10-20 \%$ of pancreatic tumours. Malignant cystic tumours of the pancreas behave similar to adenocarcinomas and thus warrant aggressive management. However, certain benign cystic neoplasms do not require operative intervention. It is, therefore, important to differentiate benign lesions from malignant lesions and from those with malignant potential. Aims: To provide an overview of the role of radiological investigations in the management of cystic neoplasms of the pancreas, with emphasis on the characteristic features of aggressive tumours. The role of different imaging modalities is discussed, and an investigative algorithm suggested. Methods: A literature review was carried out on Medline, Cochrane library, and PubMed using the MeSH terms 'pancreas' and 'cysts' to source relevant papers. Search criteria were limited to English literature, meta-analyses, systematic reviews, prospective and retrospective case series, published during or after 1998. Discussion: Each pancreatic cystic lesion has characteristic radiological findings. However, the diagnostic accuracy of individual imaging techniques is still
\end{abstract}

limited. A combination of imaging modalities is essential for preoperative diagnosis. CT complemented by endoscopic ultrasound and cyst fluid analysis appears to be the most promising investigation in diagnosing cystic neoplasms. Follow-up with serial imaging is useful for lesions of uncertain aetiology.

Copyright $\odot 2011$ S. Karger AG, Basel

\section{Introduction}

Exocrine pancreatic neoplasms include a variety of benign and malignant tumours. The majority of tumours in this group consist of pancreatic adenocarcinomas (90\%) which have a poor prognosis (5-year survival rate of 5\%) [1]. As prognosis for adenocarcinomas is so poor, it is of particular importance that there is early recognition of the pancreatic cystic tumours which have a more favourable outcome. Cystic pancreatic neoplasms account for $1-5 \%$ of all primary pancreatic tumours [2]. Nonneoplastic cysts (pseudocysts, polycystic disease, cystic fibrosis, true congenital cysts) of the pancreas (fig. 1a, b) are encountered more often than neoplastic lesions. The WHO classification (2010) of pancreatic cystic neoplasms is shown in table 1 .

\section{KARGER}

Fax +4161306 1234 E-Mail karger@karger.ch www.karger.com

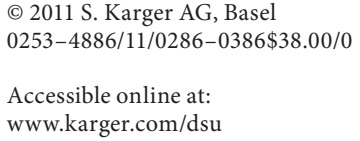

Dr. Robert A.J. Spence BEng, MB BCh BAO

Mater Hospital, Crumlin Road

Belfast BT14 6AB (UK)

Tel. +442890741211

E-Mail robert.spence@gmail.com 

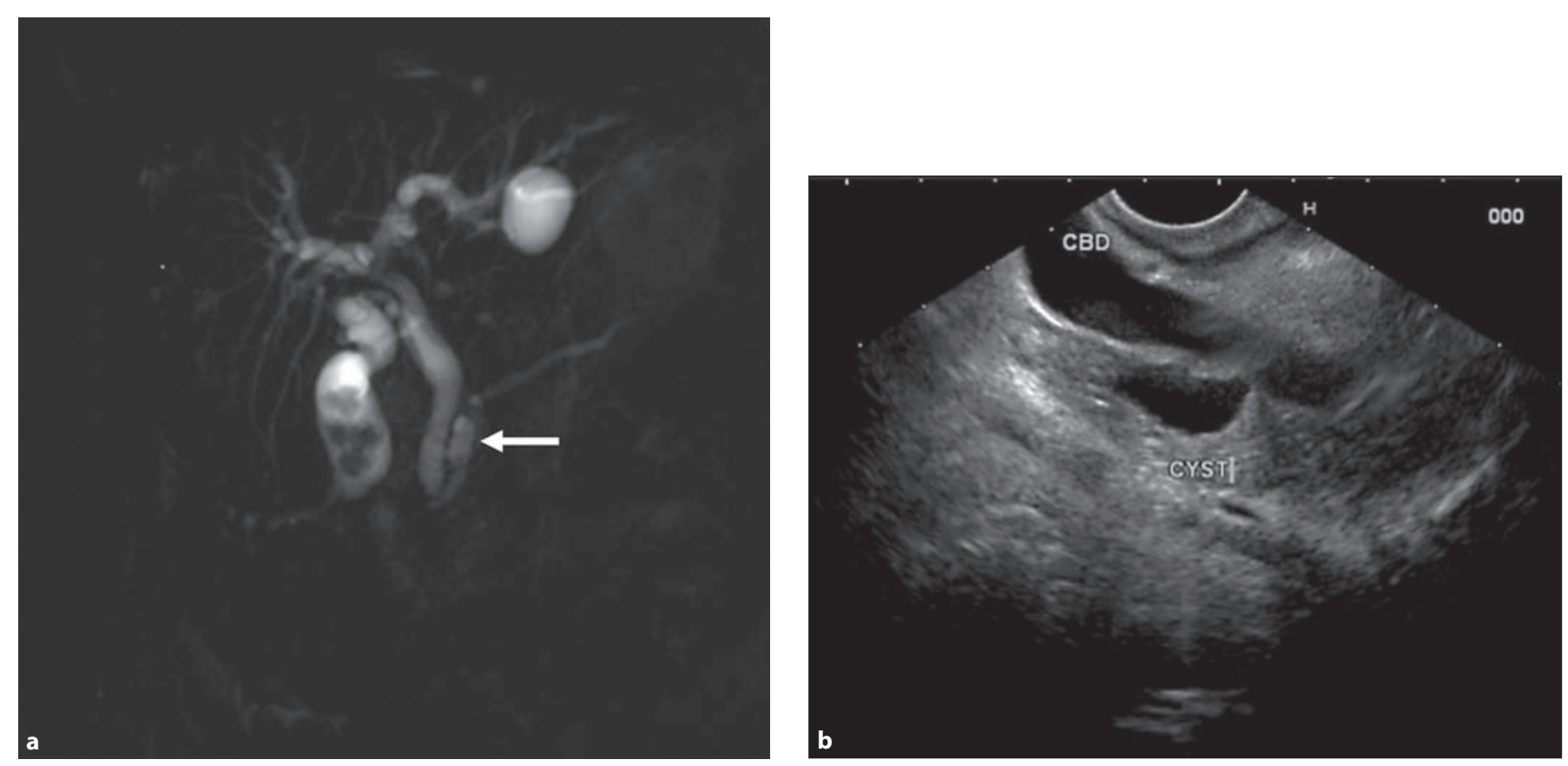

Fig. 1. a Simple cyst on MR-cholangiogram. b EUS demonstrating a simple cyst adjacent to the common bile duct $(\mathrm{CBD})$ in the head of the pancreas.

Table 1. WHO classification (2010) of pancreatic cystic neoplasms

\begin{tabular}{|c|c|c|c|}
\hline Serous cystic neoplasm & Mucinous cystic neoplasm & $\begin{array}{l}\text { Intraductal papillary mucinous } \\
\text { neoplasm }\end{array}$ & $\begin{array}{l}\text { Solid pseudopapillary } \\
\text { neoplasm }\end{array}$ \\
\hline $\begin{array}{l}\text { Serous cystadenoma } \\
\text { - Serous microcystic adenoma } \\
\text { - Serous oligocystic adenoma }\end{array}$ & Mucinous cystadenoma & $\begin{array}{l}\text { Intraductal papillary mucinous } \\
\text { adenoma }\end{array}$ & $\begin{array}{l}\text { Solid pseudopapillary } \\
\text { neoplasm }\end{array}$ \\
\hline \multirow[t]{2}{*}{ Serous cystadenocarcinoma } & $\begin{array}{l}\text { Mucinous cystic neoplasm with } \\
\text { moderate dysplasia }\end{array}$ & $\begin{array}{l}\text { Intraductal papillary mucinous } \\
\text { neoplasm with moderate dysplasia }\end{array}$ & $\begin{array}{l}\text { Solid pseudopapillary } \\
\text { carcinoma }\end{array}$ \\
\hline & $\begin{array}{l}\text { Mucinous cystadenocarcinoma } \\
\text { - Noninvasive } \\
\text { - Invasive }\end{array}$ & $\begin{array}{l}\text { Intraductal papillary mucinous carcinoma } \\
\text { - Noninvasive } \\
\text { - Invasive }\end{array}$ & \\
\hline
\end{tabular}

The management of cystic neoplasms of the pancreas has changed dramatically over recent decades due to advances in imaging, increased use of computed tomography (CT), and endoscopic techniques. However, even with these improvements in imaging, these cystic lesions are still challenging to accurately diagnose preoperatively. Over the past decade alone, there has been a 10 -fold increase in the reported incidence of cystic neoplasms, partly due to the increased use of CT imaging and endoscopic ultrasound (EUS), incidentally discovering these pancreatic cystic neoplasms [3]. This has increased the number of patients with asymptomatic cysts being referred to hepato-pancreato-biliary surgeons. There has been an associated 2 -fold increase in the number of pancreatic resections for these cystic tumours [4]. A delayed or missed diagnosis (such as mistaking a cystic tumour as a pseudocyst) can prove costly to the patient as there is the potential for malignancy with some cystic tumours.

As cystic lesions of the pancreas usually present with vague, unexplained abdominal symptoms, the patient will usually fall under the care of the general surgeon or gastroenterologist. This paper aims to provide an over- 
view of how these lesions may be investigated using different imaging modalities, highlighting current management, consequently allowing the clinician to manage these increasingly prevalent cystic lesions of the pancreas appropriately.

\section{Methodology}

A literature review was carried out on Medline, Cochrane library, and PubMed using the MeSH terms 'pancreas' and 'cysts', using Boolean Operator 'AND' to source relevant papers. Search criteria were limited to the English literature, meta-analyses, systematic reviews, prospective and retrospective cases series, published during or after 1998.

\section{Pancreatic Pseudocyst}

Any overview of cystic pancreatic neoplasms must begin with the nonneoplastic pancreatic pseudocyst, as cystic neoplasms of the pancreas are initially misdiagnosed as pseudocysts in up to $40 \%$ of cases [1]. Pseudocysts account for $75 \%$ of pancreatic 'cystic' lesions, and can develop in people of all ages $[5,6]$. Usually, pseudocysts in older patients are a consequence of alcoholic or biliary pancreatitis, whereas pseudocysts in younger patients are either hereditary or due to trauma [6]. There is equal sex distribution in the nonalcoholic pancreatitis group, but a strong male predominance in alcoholic pancreatitis patients. The incidence of pseudocysts in patients with chronic pancreatitis is $20-40 \%$ and in those with acute pancreatitis it is $2-3 \%$ [5].

Pseudocysts can occur anywhere adjacent to the pancreas (head, body, or tail), and are usually extrapancreatic. They can present in the lesser sac, retroperitoneum, between the stomach and transverse colon, stomach and liver, or in the perinephric and subdiaphragmatic spaces. They are often large $(3-20 \mathrm{~cm})$, well-demarcated cystic lesions [5]. The cyst wall is formed by inflammatory and fibrous tissue devoid of an epithelial cell lining. Pseudocysts may communicate with the pancreatic ductal system leading to complications such as pancreatic fistula and ascites [3]. Sixty percent resolve spontaneously and only persistent, symptomatic cysts need intervention.

Usually, the preceding classical history and the typical radiological features help in diagnosing a pseudocyst. However, not uncommonly, when radiological features are uncharacteristic, and when such an atypical pseudocyst is not associated with a history of clinical pancreatitis, differential diagnosis must include intraductal papil- lary mucinous neoplasm (IPMN), mucinous cystic neoplasm, and solid pseudopapillary neoplasm.

\section{Investigations}

CT, MRI and ultrasonography are all useful in the diagnosis of pseudocysts. On contrast-enhanced CT, pseudocysts are typically unilocular and may be associated with inflammatory changes in the peripancreatic fat. They characteristically have uniform, enhancing, welldefined walls. The diagnosis of pseudocyst may be further supported by atrophy, or calcification of the pancreatic parenchyma [7].

On MRI, pseudocysts demonstrate uniform low signal intensity on T1-weighted images and high signal intensity on T2-weighted images. A nonhomogeneous appearance of the cyst on CT or MRI usually represents haemorrhage or necrotic debris (fig. 2a). Communication of the pseudocyst with the pancreatic duct may be seen on endoscopic retrograde cholangiopancreatography (ERCP) or magnetic resonance cholangiopancreatography (MRCP). It should be noted that cyst communication with the pancreatic duct can also be seen with IPMN.

Endoscopic ultrasound (fig. 2b) and aspiration of the cyst is helpful in the investigation of a pseudocyst where diagnostic doubt remains after ultrasound, CT, and MR. The aspirated fluid consists of granular debris, lipid droplets, and inflammatory cells, such as neutrophils, macrophages, and plasma cells. The fluid has a characteristic high amylase, low lipase levels, and low viscosity [8].

\section{Management}

Occasionally, diagnosis of a pancreatic pseudocyst can be difficult. When there is a unilocular cyst with a lobulated contour located adjacent to the head of the pancreas, a serous cystadenoma should be considered. Asymptomatic, thin-walled unilocular cysts can be monitored with CT or MR imaging, especially when they are small [9]. As up to $60 \%$ of pseudocysts will resolve without intervention, repeated imaging of uncertain lesions may be warranted to help differentiate between pseudocysts and cystic neoplasms. For those symptomatic pseudocysts which do not resolve, there are a number of techniques used to drain the pseudocyst: endoscopically, percutaneously, or surgically. Currently, endoscopic drainage is the preferred technique, as shown in a recent large study, which was effective in $89 \%$ of patients with complete drainage of the pseudocyst, with only a $12 \%$ recurrence rate, and minor complications (bleeding, infection, mild pancreatitis) in $11 \%$ of patients [10]. This compares favourably to both percutaneous drainage ( $21 \%$ effective resolution of pseu- 

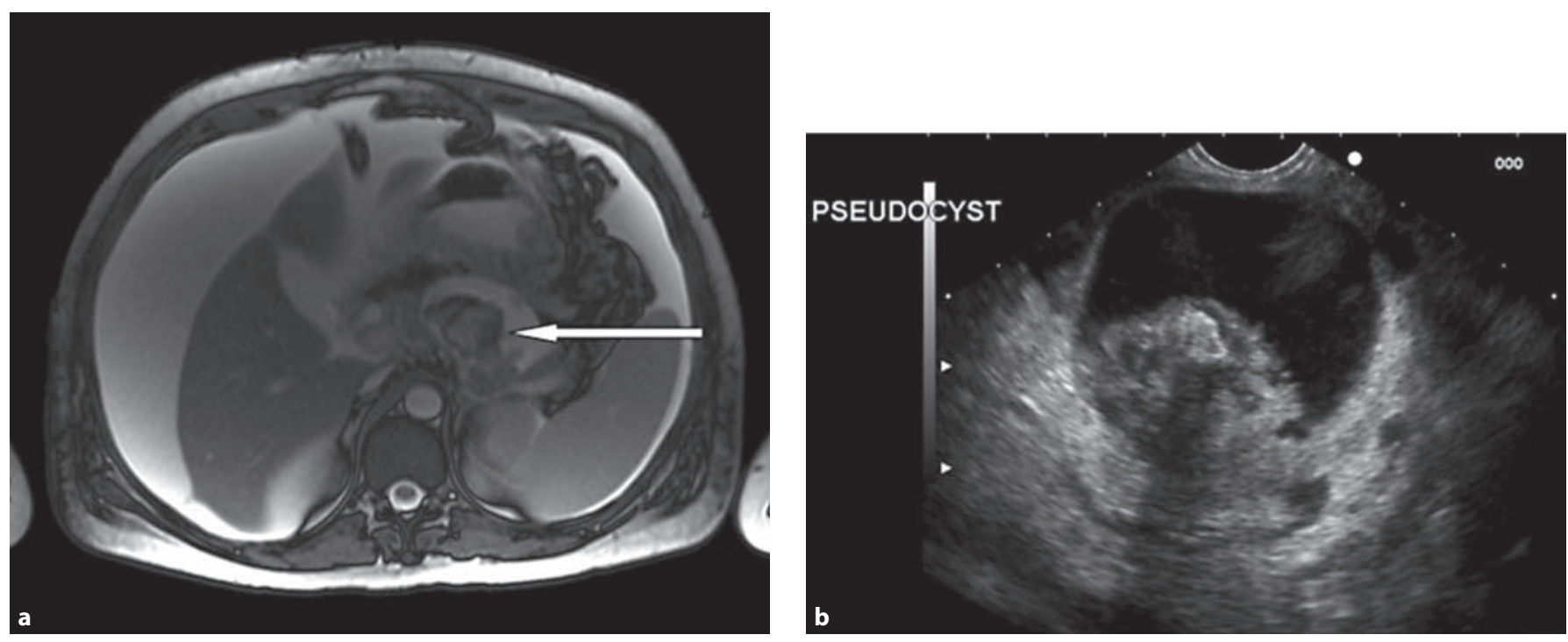

Fig. 2. a T2-weighted image on MRI demonstrating a pseudocyst in the body of pancreas, posterior to the stomach. Note the cyst is filled with debris. b Large pseudocyst filled with debris on EUS.

docyst) and open/laparoscopic surgical management. Surgical management is usually effective, but is associated with a higher rate of complications (12-35\%) [11-13].

\section{Pancreatic Serous Cystic Neoplasms}

Serous cystic neoplasms represent approximately $30 \%$ of primary cystic neoplasms of the pancreas, with the largest subset being the serous cystadenoma [14]. Malignant serous cystadenocarcinomas account for only $1-3 \%$ of serous tumours of the pancreas $[15,16]$.

Serous cystadenomas are benign cystic tumours of the pancreas that arise from the acinar cells [17]. They are twice as common in men as in women, with a mean patient age of 70 years [5]. Most patients present with vague symptoms such as epigastric pain or fullness, nausea, vomiting, dyspepsia, fever, melaena, or weight loss. However, up to $30 \%$ of patients can be asymptomatic, with the tumour found incidentally on imaging $[4,17]$. The average rate of growth of a serous cystadenoma is thought to be $0.5-0.6 \mathrm{~cm} /$ year [3].

Most serous cystadenomas are firm, well-circumscribed, multilobulated tumours. Fifty percent of these lesions are located within the head of pancreas $[4,5]$. The size of the lesions can vary from a diameter of $1-20 \mathrm{~cm}$, with a mean of $6 \mathrm{~cm}$. They are microcystic and have a simple cuboidal epithelial lining on histology [4]. The numerous small cysts, filled with clear fluid, separated by the dense, lace-like matrix of fibrous septa give the tumour a 'sponge-like' or honeycomb appearance. A central stellate scar (present in 13-18\% of cases [1]) caused by calcification of the central fibrous stroma creates the pathognomonic 'starburst' appearance. They are usually single; however, multifocal lesions may be associated with Von Hippel-Lindau disease [5].

\section{Investigations}

With improvements in imaging techniques, serous cystadenomas can be distinguished by their clinical presentation and imaging characteristics in over $90 \%$ of patients [18]. On nonenhanced CT, serous cystadenomas are of varying density, with margins of the lesion ranging from poorly defined to a thin well-defined capsule [1]. CT imaging with contrast demonstrates enhancement of septa, delineating small cysts in a honeycomb pattern [7]. There may be dystrophic calcification within a central scar and capsular enhancement. This may be associated with common bile duct dilatation and atrophy of the pancreas distal to the tumour [17].

MRI demonstrates the honeycomb appearance with low signal intensity on T1-weighted images and high intensity on T2-weighted images $[1,9]$. The tumour may have external lobulation and is well defined with no invasion of 

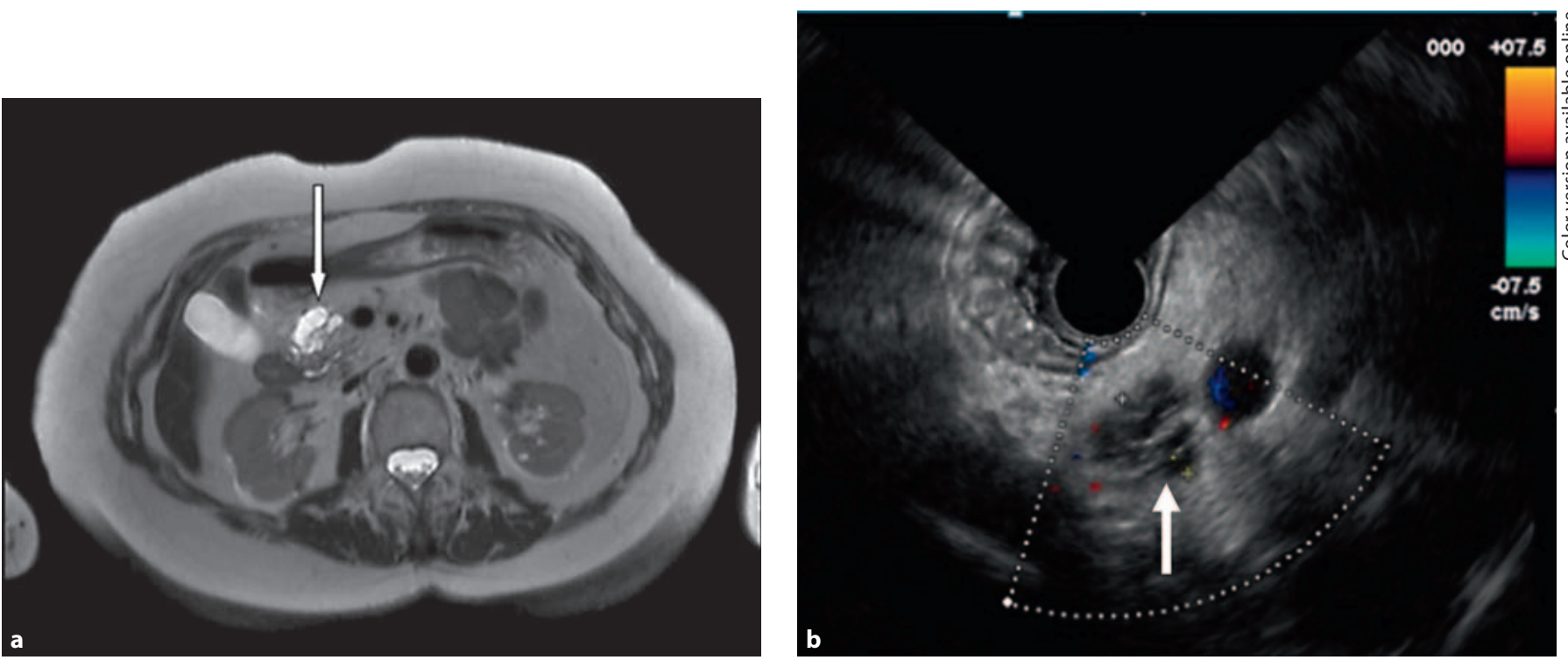

Fig. 3. Microcystic serous cystadenoma in the head of pancreas on MRI (a) and on EUS (b). Note the honeycomb pattern due to multiple septa within the lesion on EUS.

fat or adjacent organs. Small cysts and septa separating different segments are seen as high signal 'grape-like' clusters on T2-weighted images (fig. 3a). Any haemorrhage within the locules causes high signal intensity on T1-weighted images. However, unlike CT, the key pathognomonic central calcifications are rarely visualised with MR imaging [1].

The CT appearance of serous cystadenomas and mucinous neoplasms can be similar and difficult to distinguish. EUS can help distinguish these lesions using morphological features, and also permits aspiration of cyst contents. The characteristic honeycomb pattern in serous cystadenomas may also be visualised with EUS (fig. 3b). Absence of mucin in the fluid favours the diagnosis of serous cystadenoma.

\section{Management}

The characteristic findings of stromal hypervascularity with predominance of small grape-like cystic areas, combined with an indolent course, lack of local invasion or metastases and appropriate clinical features, allow this diagnosis to be made noninvasively [18]. Treatment for symptomatic patients with serous cystadenoma is surgical resection. If the tumour is located in the head of the pancreas, a pancreaticoduodenectomy should be performed. Distal pancreatectomy should be carried out for tumours in the body and tail. However, there are other options available to the surgeon. If the tumour is located in the neck or proximal body of the pancreas, a local resection may be performed with less morbidity, including diabetes [4]. Age is a factor in management for asymptomatic patients. As many patients are elderly when diagnosed, these patients can be managed conservatively. However, if an asymptomatic lesion is discovered in a younger patient, the decision is more complex. The tumours can increase in size and later become symptomatic. This was demonstrated by Tseng et al. [4], who showed that the likelihood of symptoms increases with the size of the tumour ( $22 \%$ with tumours $<4 \mathrm{~cm}$ in diameter versus $77 \%$ with tumours $\geq 4 \mathrm{~cm}$ ). If the patient is treated conservatively, it is important to be confident that this notoriously difficult tumour to diagnose is indeed a benign serous cystadenoma and not a potentially malignant mucinous cystic neoplasm. Discussion at a hepatobiliary multidisciplinary meeting including gastroenterologists, surgeons, radiologists, and pathologists is essential.

\section{Pancreatic Mucinous Cystadenoma and Cystadenocarcinoma}

Mucinous tumours represent approximately $2 \%$ of all pancreatic neoplasms and one third of cystic neoplasms. They are typically large multilocular cysts, characterised 

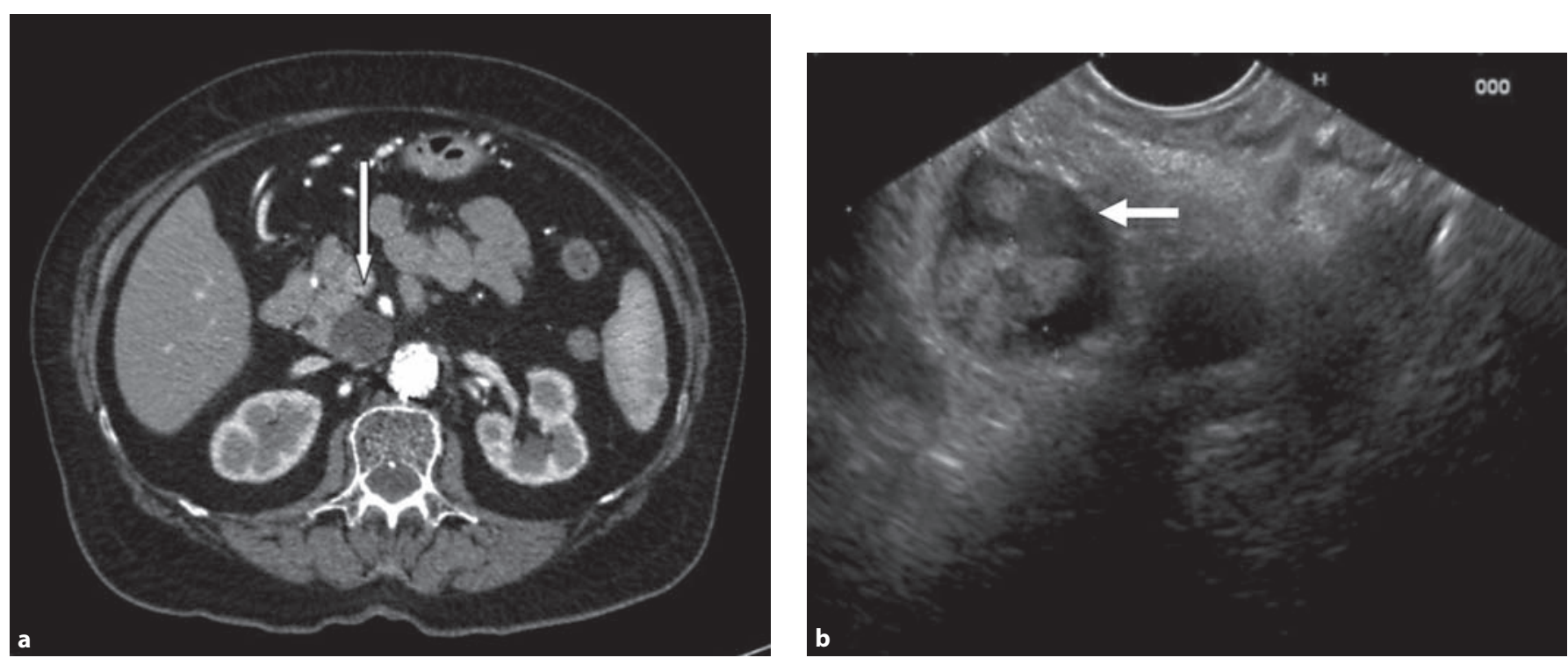

Fig. 4. Mucinous cystadenoma: a CT of the pancreas demonstrating a unilocular cystic lesion in the uncinate process. b As shown by EUS, the cyst is filled with solid nodules.

by mucin-producing columnar epithelium, resting on a fibrous ovarian-type stroma [3]. These mucinous lesions can be benign mucinous cystadenomas, borderline with malignant potential, or malignant (carcinoma in situ or invasive carcinoma). Mucinous neoplasms have a high female preponderance (female:male $=9: 1$ ), and usually present during middle age (mean age at diagnosis: 48 years) $[3,19]$. Most of these patients present with vague abdominal symptoms including epigastric pain (64\%), mass $(25 \%)$, or a sense of abdominal fullness. Less commonly, the patients suffer from nausea, vomiting, diarrhoea, anorexia, and weight loss. In the case of mucinous cystadenocarcinoma, jaundice is a feature in $32 \%$ of patients due to the tumour being located in the head of pancreas and impinging upon the common bile duct $[1,5]$.

The majority (70-90\%) of mucinous cystic neoplasms arise in the body or tail of pancreas, with only $10-30 \%$ being located in the head of pancreas [3,5]. Mucinous cystic neoplasms are usually large - the mean diameter ranges from 7 to $10 \mathrm{~cm}$ [5]. They usually contain several cystic areas that are $>2 \mathrm{~cm}$ in diameter, but can also present as a single macrocystic lesion. The cyst wall is typically $1-2 \mathrm{~mm}$ thick and contains calcification in up to $30 \%$. The epithelium of a mucinous cystadenoma demonstrates a wide range of architecture ranging from a uniform simple epithelium to complex papillary folds which form a cribriform pattern. The walls may be irregular or contain solid papillary excrescences, both of which are suggestive of malignancy [1]. Although mucinous cystic neoplasms do not communicate with the pancreatic duct, they can cause partial obstruction of the duct $[3,8]$.

\section{Investigations}

The classic imaging appearance of a mucinous cystic neoplasm is that of a large cystic mass with septa in the pancreatic body or tail. On CT imaging, mucinous cystic tumours appear as 'near water' density, unilocular or multilocular lesions with enhancement of thin internal septa and peripheral wall (fig. 4a). The presence of solid component, calcification, a thick wall ( $>2 \mathrm{~mm})$, and septations indicate a high (95\%) risk of malignancy [20]. Peripheral 'eggshell' calcification, present in $<20 \%$ of mucinous cystic neoplasms, is specific for a mucinous cystic neoplasm and is highly predictive for malignancy [7, 9, 18]. Mural nodules and papillary excrescences into the cysts are more common in cystadenocarcinomas [5]. Features of chronic pancreatitis such as pancreatic atrophy, duct dilatation or calcification may accompany the tumour. Local invasion is associated with obliteration of the fat planes and margins of adjacent organs.

With MR imaging, T1-weighted images show variable signal intensity based on fluid content. Simple fluid has a hypodense signal intensity, whereas proteinaceous or haemorrhagic material demonstrates hyperdense signal intensity. With T2-weighted images, cysts can appear hyperdense with hypodense internal septations [17]. 
On EUS, mucinous cystic tumours appear as hypoechoic complex cysts, often with solid components visible (fig. 4b). This is in contrast to a serous cystadenoma which displays the characteristic honeycomb pattern. EUS can also reveal characteristics that are predictive of malignancy, such as local invasion, regional nodal disease, adjacent mass, and the presence of vascular collaterals [21].

\section{Management}

Surgical excision is indicated for all mucinous cystic neoplasms. As a negative fine needle aspiration cytology (FNAC) cannot rule out malignancy, all suspected mucinous cystic neoplasms must be resected. If the diagnosis is uncertain, and the patient is asymptomatic, observation of cysts less than $3 \mathrm{~cm}$ may be justified given that the risk of malignancy is equal, or less than, the operative mortality [3]. This was shown in three recent studies which demonstrated a $3 \%$ malignancy risk for mucinous cystic neoplasms with a diameter less than $3 \mathrm{~cm}$, which they noted was approximately the same as the risk of mortality from resection [22-24].

As most mucinous cystic neoplasms are located in the body and tail of pancreas, they require distal pancreatectomy. For larger neoplasms, or whenever there is suspicion of carcinoma, a splenectomy should also be carried out to remove the potentially involved nodal basin. A pancreaticoduodenectomy is indicated for any tumours located in the head of pancreas. Complete resection produces an almost $100 \%$ cure rate for lesions, if they have no evidence of transmural invasion. For patients with invasive mucinous cystadenocarcinoma, the 5 -year survival decreases to $30-40 \%$, which still compares favourably with the more common ductal adenocarcinoma of the pancreas [4].

\section{Pancreatic IPMN}

IPMNs represent approximately $1 \%$ of all pancreatic neoplasms and $25 \%$ of cystic neoplasms. They have been diagnosed with increasing frequency since their initial description in Japan as a 'mucin-producing' cancer of the pancreas [25].

IPMN is defined pathologically as a neoplastic proliferation of mucin-producing cells, lining the main or secondary branch pancreatic ducts. Main-duct IPMNs are characterised by involvement of the main pancreatic duct with, or without, associated involvement of the branch ducts (combined IPMNs). These neoplasms produce considerable amounts of mucin, which distends the local duct system, causing ductal ectasia, and creating a multiloculated mass. Branch-duct IPMNs involve the side branches of the pancreatic ductal system and appear as cystic lesions communicating with a nondilated main pancreatic duct. Seventy-five percent of main-duct IPMNs are located in the proximal portion of the pancreas and tend to spread to the remaining main pancreatic duct. Branch-duct IPMNs are more commonly found in the uncinate process, but can also be seen in the head, neck, or distal pancreas. A common finding is multifocal involvement of the pancreas with two or more branchduct IPMNs $[3,26]$.

IPMNs can be categorised histologically into benign (adenoma), borderline, and malignant (in situ carcinoma and invasive carcinoma). There is an equal distribution between males and females. A study in 2004, of 140 patients, noted that $25 \%$ of patients with IPMN were asymptomatic when diagnosed. When symptoms were present, the most common for main-duct IPMN were abdominal pain $(65 \%)$, weight loss $(44 \%)$, acute pancreatitis $(23 \%)$, jaundice (17\%), diabetes (12\%), and steatorrhoea (6.5\%) [27]. Branch-duct IPMNs can present with the typical symptoms outlined above; however, a large proportion are asymptomatic. These asymptomatic neoplasms are usually detected incidentally through radiological investigation of other symptoms [26].

The mean age for presentation of an IPMN is 68 years. However, it must be noted that the mean age of patients with main-duct IPMN malignant tumours is 6.4 years older than the age of those with borderline lesions. This supports the current view that IPMNs progress with time towards malignancy [4].

Recent evidence suggests the malignancy rate of mainduct IPMN has been found to be significantly higher (4992\%) than that of branch-duct IPMNs (6-46\%) [28-34]. It is, therefore, of importance to distinguish if the IPMN is of main-duct or branch-duct origin preoperatively to guide optimum management strategy. Larger tumour size $(>30 \mathrm{~mm})$, thick cyst wall, proximal location, presence of mural nodules, protruding lesions in dilated branch ducts and mucin leakage from a patulous ampulla are further predictors of malignancy for IPMN [30].

\section{Investigations}

On nonenhanced CT, a main-duct IPMN is demonstrated as a dilated and tortuous main pancreatic duct. A branch-duct IPMN appears as a lobulated cystic lesion with characteristic grape-like clusters or 'tubes and arcs' appearance, limited to segmental ducts. This results in a single cystic mass within an otherwise normal ductal sys- 

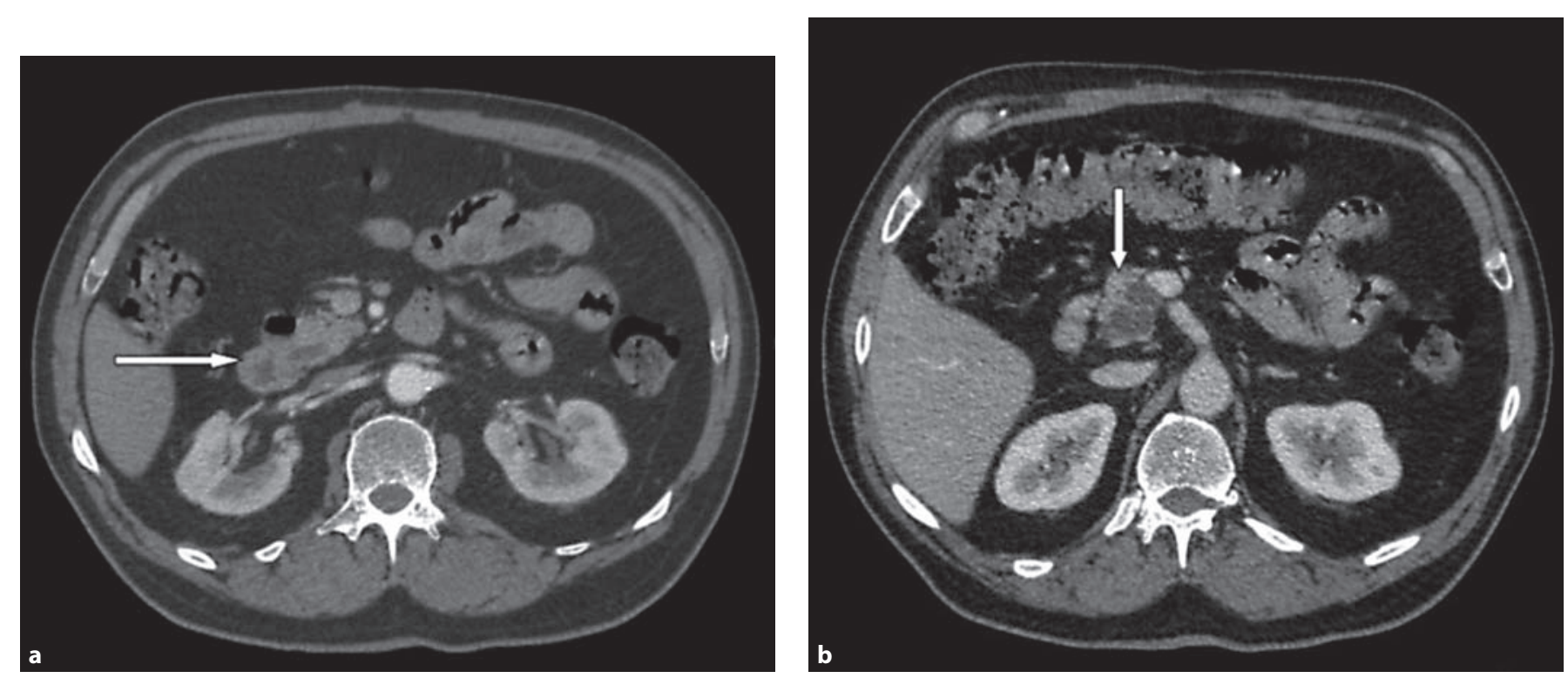

Fig. 5. a CT of the head and uncinate process of pancreas demonstrating a side branch IPMN. b Note the typical fish mouth appearance due to bulging of ampulla.

tem. For combined IPMNs, cystic lesions are found typically in the uncinate process. They have a characteristically lobular appearance with multiple intercommunicating cysts, and a grossly dilated main pancreatic duct $[1,17]$. With enhanced CT, thin irregular, peripheral ring-enhancing multicystic lesions may be visible with branch-duct lesions. There may also be a bulging ampulla with thin rim enhancement [17].

With T1-weighted MR imaging in the axial plane, low intensity branch-duct cysts with main-duct dilatation can be visualised. Clustered cystic lesions with thin septa can be seen in the coronal plane. T2-weighted MR imaging is more useful in evaluating IPMNs. This shows high signal intensity of the dilated main-duct, and can demonstrate a cystic mass associated with IPMNs of the branch-duct subtype $[1,17]$. MRCP can show not only the main pancreatic duct, but also the cystic lesion, which is not always possible with ERCP, as shown by Koito et al. [35] MRCP is particularly useful in the characterisation of a single or multifocal branch-duct IPMN, as it can demonstrate communication between the main-duct and the cyst.

ERCP is especially helpful in demonstrating the classical triad of Ohashi [36]: bulging ampulla of Vater (fig. 5a, b), mucin secretion, and dilated pancreatic duct. It is possible to obtain real-time visualisation of the patulous ampulla with increased mucus production. With main-duct IPMN, injection of contrast demonstrates diffuse dilata- tion of the main-duct, accompanied by intraluminal filling defects representing small papillary projections. However, due to thick mucus blocking the contrast, ERCP can occasionally fail to demonstrate the entire ductal system. A branch-duct IPMN will show ectasia of one or a group of side branches, usually located in the uncinate process $[1,5]$. Both ERCP and MRCP can be used for diagnosing mucin-producing cystic tumours (mucinous cystic neoplasms and IPMNs).

\section{Management}

According to the International Association of Pancreatology guidelines for the management of IPMNs, all suspected main-duct and combined IPMNs should be resected due to the high risk of malignancy. Asymptomatic patients should undergo resection if fit, especially as there is no current method of distinguishing benign and malignant IPMN preoperatively. It must be noted that preoperative imaging studies may only show a segmental dilatation of the main pancreatic duct. Dilatation can occur both proximally and distally to the tumour, making localisation of the tumour challenging. Typical resections for main-duct and combined IPMNs include pancreaticoduodenectomy, distal pancreatectomy, and total pancreatectomy depending on the site of the tumour. Lymph node dissection must also be performed due to the high risk of invasion $[26,30]$. 
Table 2. Imaging and cyst fluid characteristics of cystic lesions of pancreas

\begin{tabular}{lll}
\hline Pancreatic cystic lesion & Imaging characteristics & Fluid analysis \\
\hline Pseudocyst & Usually associated with pancreatitis & $\downarrow$ Viscosity \\
& Unilocular & $\downarrow$ Lipase \\
& Uniform, enhancing, well-defined walls & $\uparrow$ Amylase \\
& Peripancreatic inflammation & $\leftrightarrow$ CEA levels \\
& May communicate with pancreatic duct & \\
\hline Serous cystic lesion & Microcystic & $\downarrow$ Viscosity \\
& Honeycomb pattern of microlacunae & $\downarrow$ CEA \\
& Stellate scar & $\downarrow$ CA19-9 \\
& Central calcifications - starburst appearance & $\downarrow$ Amylase \\
\hline Mucinous cystic lesion & Typically unilocular but can be multilocular & $\uparrow$ Viscosity \\
& Body/tail of the pancreas & $\uparrow$ CEA \\
& Macrocystic & $\uparrow$ CA19-9 \\
& No duct communication & $\downarrow$ Amylase \\
& Peripheral 'eggshell’ calcifications & \\
\hline IPMN & Macrocystic & $\uparrow$ Viscosity \\
& Ductal involvement & $\uparrow$ CEA \\
& - Main duct: dilatated, tortuous main pancreatic duct & $\uparrow$ Amylase \\
& - Branch duct: lobulated lesion with grape-like clusters & \\
\hline
\end{tabular}

Branch-duct IPMNs are associated with malignancy in $6-46 \%$ of cases. Signs of malignancy in branch-duct IPMNs include: a symptomatic patient; lesion size $>3 \mathrm{~cm}$; or the presence of mural nodules. If any of these features are present, the lesion must be resected in a fit patient. However, if the patient is asymptomatic, with a lesion size $<3 \mathrm{~cm}$, and without mural nodules, the patient may be managed with careful observation. If there is suspicion of malignancy of a branch-duct IPMN, a total pancreatectomy will be required if the disease is multifocal [26]. A more selective approach can be used if the disease is not widespread [37].

\section{Role of EUS and Cyst Fluid Analysis in the Management of Cystic Lesions of Pancreas}

The use of transabdominal sonography is limited in the evaluation of pancreatic lesions as images can be poor due to overlying bowel and unfavourable body habitus (adipose tissue in the abdominal wall). The role of newer techniques of examination such as contrast-enhanced ultrasound using microbubble technology is being investigated in the assessment of vascular involvement of tumours and the presence of malignant lymph nodes [14].

EUS provides better delineation of the pancreas. It helps to assess the characteristics of the lesion, pancre- atic ductal anatomy, pancreatic parenchyma, peripancreatic invasion of tumour, and the presence of lymphadenopathy. The presence of a solid component within the cystic lesion, thick wall, multiple septa, invasion outside the pancreas, pancreatic ductal obstruction and lymphadenopathy indicate malignancy $[9,38]$. However, EUS is operator dependent with a low sensitivity of $50-60 \%[1$, 38]. Therefore, it is still considered a complementary procedure to CT or MR imaging.

EUS offers guidance for fine needle aspiration (FNA) of suspicious cystic lesions which has been demonstrated to be as accurate as CT-guided FNA; for pancreatic lesions of $<3 \mathrm{~cm}$, EUS-guided FNA has been shown to be more accurate than CT-guided FNA [39]. With CT or EUS guidance, fine needle aspiration allows fluid to be obtained for cytology and measurement of tumour markers, viscosity, and amylase. EUS-FNA is highly specific in the diagnosis of cystic pancreatic lesions but sensitivity is only $20-50 \%$, and is only useful when malignant cells are demonstrated [40]. Fluid analysis of the cyst for tumour markers, viscosity, and amylase has proved to be more useful than cytology in distinguishing malignant cystic lesions of the pancreas. Of the several intracystic proteins studied (CA 19-9, CEA, CA 125 and CA 72-4), the level of CEA is most accurate and is often used in routine practice.

Brugge et al. [40] determined that cyst fluid analysis of CEA level $(>192 \mathrm{ng} / \mathrm{ml})$ is the most accurate investiga- 


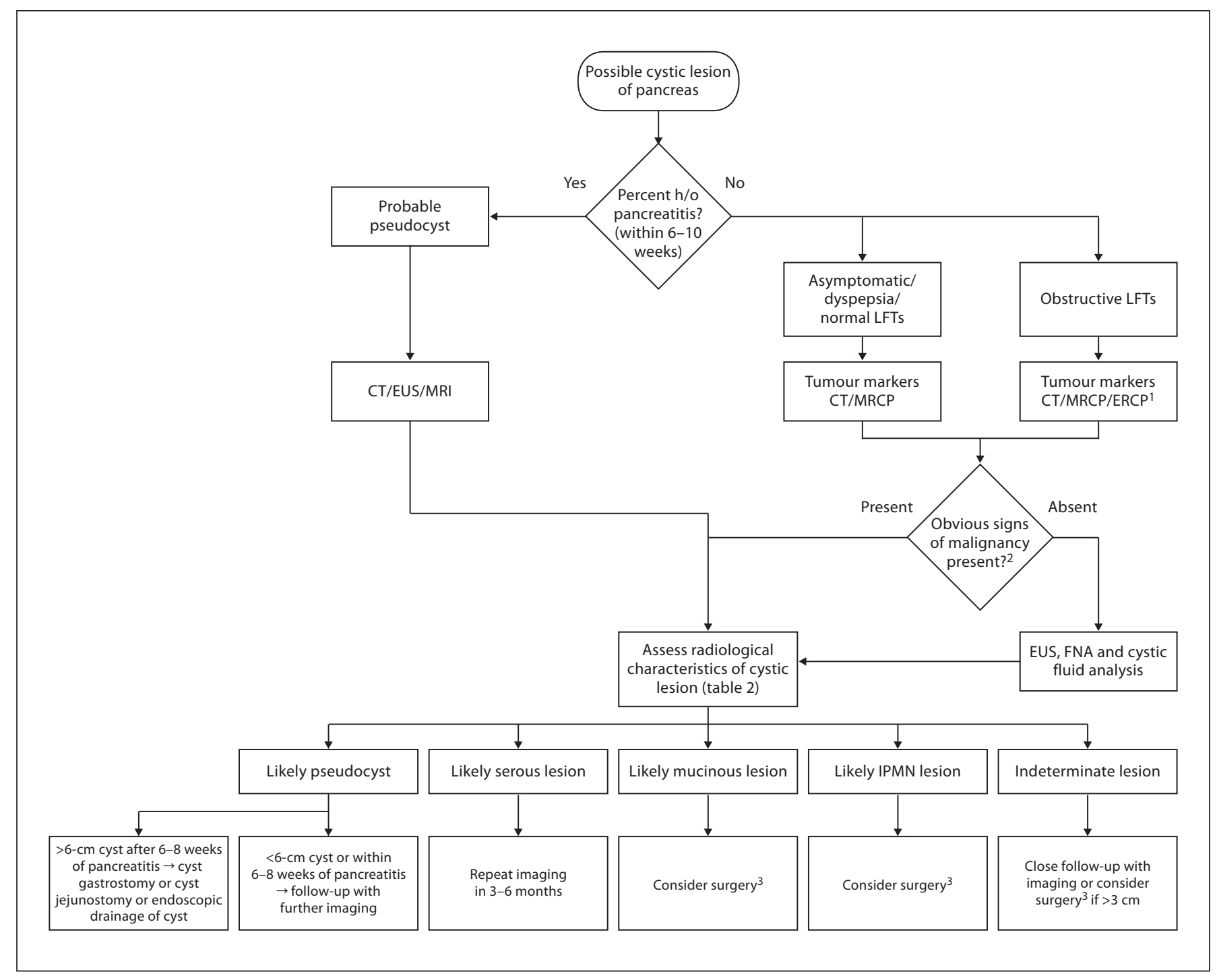

Fig. 6. Flowchart of the investigation and management of a cystic lesion of the pancreas. h/o = History of; LFT = Liver Function Tests. ${ }^{1}$ Biliary stent could be considered for obstruction and biopsies taken. ${ }^{2}$ Significant lymphadenopathy, local invasion. ${ }^{3} \mathrm{~Pa}$ -

tion in diagnosing mucinous cystic neoplasms. It has been shown that high viscosity $(>1.6)$ and CEA of $>6,000$ $\mathrm{ng} / \mathrm{ml}$, has a $94.3 \%$ accuracy rate in diagnosing mucinous cystadenocarcinomas. When CEA levels are $<6,000$ $\mathrm{ng} / \mathrm{ml}$ with a high viscosity $(>1.6)$, there is an accuracy rate of $97.2 \%$ in predicting mucinous cystadenomas. It was also demonstrated when cystic fluid analysis had viscosity $<1.6$ and lipase $<6,000 \mathrm{U} / 1$, serous cystadenomas were predicted with $100 \%$ accuracy [8]. Cyst fluid analysis of IPMNs is similar to mucinous cystic neoplasms - high in viscosity and the tumour marker CEA, tient's age and comorbidities determine suitability for surgery. Presence of portal vein thrombosis and liver metastasis may indicate inoperability.

indicating malignancy. However, unlike mucinous cystic neoplasms, IPMNs contain high amylase due to communication of the cyst with the main pancreatic duct [41].

\section{Role of PET CT}

The routine use of whole body PET imaging does not provide any benefit compared to contemporary crosssectional imaging in distinguishing benign from malig- 
nant pancreatic cysts. It has a low sensitivity (57\%) and specificity $(85 \%)$ in identifying malignant pathology in pancreatic cystic lesions [42].

\section{Management of a Patient with a Cystic Lesion of Pancreas}

Although the classical radiological and pathological features of cystic lesions of pancreas are well described in the literature, it is often difficult to make a definite preoperative diagnosis. Despite of the characteristic CT and MR findings, overall diagnostic accuracy of these investigations ranges from 20 to $80 \%$. Procacci et al. [43] reported an accurate preoperative diagnosis of tumour type on CT in only $20 \%$ of those with serous cystadenoma, $30 \%$ of those with mucinous cystadenoma, and 30\% of those with mucinous cystadenocarcinoma. Low accuracy rates of radiological investigations of these cystic lesions can make the diagnosis and clinical decision challenging. It is, therefore, useful to correlate the radiological findings with cyst fluid analysis (table 2). We have created an algorithm to assist the general surgeon in the investigation and management of a cystic lesion of the pancreas (fig. 6). Surgical excision is usually the treatment of choice for mucin-producing cystic tumours (mucinous cystadenoma, mucinous cystadenocarcinoma or IPMN). Lesions greater than $3 \mathrm{~cm}$ are likely to be premalignant and require an aggressive surgical approach. Local invasion of the tumour (portal vein thrombosis, significant lymphadenopathy) and presence of distant metastases indicate inoperability. Patient's age and comorbidities need to be taken into account when surgical resection is considered. An elderly patient with an indeterminate lesion or a likely serous lesion is better followed up (especially if they have significant comorbidity), whereas a younger patient with a mucin-producing lesion will benefit from an aggressive surgical resection.

\section{Conclusion}

Although cystic lesions have characteristic radiological findings, the accuracy of individual imaging modalities is limited. CT remains the primary investigative modality in the diagnosis and staging of the pancreatic lesion. MR provides better resolution than CT for evaluating small cystic lesions less than $3 \mathrm{~cm}$, as well as defining ductal communication and cyst morphology. EUS is an excellent complementary investigation to CT and MR for imaging and allows aspiration of the cystic lesion for fluid analysis (CEA, CA 19-9, and amylase). If diagnosis is uncertain and the patient is asymptomatic, observation of cysts $<3 \mathrm{~cm}$ (which have no malignant features on imaging), may be justified given that the risk of malignancy is equal or less than, the operative mortality [3].These 'uncertain' lesions can be followed-up by serial imaging. If changes occur, or size increases, then discussion at a hepatobiliary multidisciplinary team meeting is essential to determine if resectional surgery is required.

\section{References}

1 Visser BC, Muthusamay VR, Mulvihill SJ, Coakley F: Diagnostic imaging of cystic pancreatic neoplasms. Surg Oncol 2004;13:27-39.

-2 Visser BC, Muthusamy VR, Yeh BM, Coakley FV, Way LW: Diagnostic evaluation of cystic pancreatic lesions. HPB (Oxford) 2008;10:63-69.

3 Carpizo DR, Allen PJ, Brennan MF: Current management of cystic neoplasms of the pancreas. Surgeon 2008;6:298-307.

4 Tseng JF, Castillo CF, Warshaw AL: Cystic neoplasms of the pancreas; in Blumgart LH, Fong Y (eds): Surgery of the Liver and Bilary Tract, 3rd ed. 858-866, WB Saunders, 2000.

5 Degan L, Wiesner W, Beglinger C: Cystic and solid lesions of the pancreas; in Tytgat G (ed): Clinical Gastroenterology. 91-103, Elsevier, 2007.
6 Kaosmahl M, Kloppel G: Pancreatic cystic lesions and neoplasms; in Johnson CD, Imrie CW (eds): Pancreatic Disease. 133-143, Springer, 2004.

$\checkmark 7$ Brugge WR, Lauwers GY, Sahani D, Fernandez-del Castillo C, Warshaw AL: Cystic neoplasms of the pancreas. N Engl J Med 2004; 351:1218-1226.

$>8$ Linder JD, Geenen JE, Catalano MF: Cyst fluid analysis obtained by EUS-guided FNA in the evaluation of discrete cystic neoplasms of the pancreas: a prospective single-center experience. Gastrointest Endosc 2006;64: 697-702

$\checkmark 9$ Sahani DV, Kadavigere R, Saokar A, Fernandez-del Castillo C, Brugge WR, Hahn PF: Cystic pancreatic lesions: a simple imaging-based classification system for guiding management. Radiographics 2005;25: 1471-1484.
10 Ahn JY, Seo DW, Eum J, Song TJ, Moon SH, Park do H, et al: Single-step EUS-guided transmural drainage of pancreatic pseudocysts: analysis of technical feasibility, efficacy, and safety. Gut Liver 2010;4:524-529.

11 Bergman S, Melvin WS: Operative and nonoperative management of pancreatic pseudocysts. Surg Clin North Am 2007;87:14471460, ix.

12 Usatoff V, Brancatisano R, Williamson RC: Operative treatment of pseudocysts in patients with chronic pancreatitis. Br J Surg 2000;87:1494-1499.

13 Gumaste VV, Aron J: Pseudocyst management: endoscopic drainage and other emerging techniques. J Clin Gastroenterol 2010;44: 326-331.

14 Kinney T: Evidence-based imaging of pancreatic malignancies. Surg Clin North Am 2010;90:235-249. 
-15 Abe H, Kubota K, Mori M, Miki K, Minagawa M, Noie T, et al: Serous cystadenoma of the pancreas with invasive growth: benign or malignant? Am J Gastroenterol 1998;93: 1963-1966.

-16 Strobel O, Z'Graggen K, Schmitz-Winnenthal FH, Friess H, Kappeler A, Zimmermann A, et al: Risk of malignancy in serous cystic neoplasms of the pancreas. Digestion 2003; 68:24-33.

17 Federle MP, Fishman E, Jeffrey RB, Anne VS: Pocket Radiologist. Abdominal. Top 100 Diagnoses. 81-83, Elsevier Science, 2004.

-18 Sarr MG, Kendrick ML, Nagorney DM, Thompson GB, Farley DR, Farnell MB: Cystic neoplasms of the pancreas: benign to malignant epithelial neoplasms. Surg Clin North Am 2001;81:497-509.

$\checkmark 19$ Wilentz RE, Albores-Saavedra J, Hruban RH: Mucinous cystic neoplasms of the pancreas. Semin Diagn Pathol 2000;17:31-42.

20 Procacci C, Carbognin G, Accordini S, Biasiutti C, Guarise A, Lombardo F, et al: CT features of malignant mucinous cystic tumors of the pancreas. Eur Radiol 2001;11: 1626-1630.

-21 Hernandez LV, Mishra G, Forsmark C, Draganov PV, Petersen JM, Hochwald SN, et al: Role of endoscopic ultrasound (EUS) and EUS-guided fine needle aspiration in the diagnosis and treatment of cystic lesions of the pancreas. Pancreas 2002;25:222-228.

-22 Goh BK, Tan YM, Chung YF, Chow PK, Cheow PC, Wong WK, et al: A review of mucinous cystic neoplasms of the pancreas defined by ovarian-type stroma: clinicopathological features of 344 patients. World J Surg 2006;30:2236-2245.

-23 Allen PJ, Jaques DP, D’Angelica M, Bowne WB, Conlon KC, Brennan MF: Cystic lesions of the pancreas: selection criteria for operative and nonoperative management in 209 patients. J Gastrointest Surg 2003;7:970-977.

-24 Allen PJ, D’Angelica M, Gonen M, Jaques DP, Coit DG, Jarnagin WR, et al: A selective approach to the resection of cystic lesions of the pancreas: results from 539 consecutive patients. Ann Surg 2006;244:572-582.

-25 Yamaguchi K, Tanaka M: Intraductal papillary-mucinous tumor of the pancreas: a historical review of the nomenclature and recent controversy. Pancreas 2001;23:12-19.
26 Abu Hilal M, Peiris L, Salvia R: Intraductal papillary mucinous neoplasms of the pancreas; in Taylor I, Johnson C (eds): Recent Advances in Surgery. 83-97, Royal Society of Medicine Press Ltd, 2008.

27 Salvia R, Fernandez-del Castillo C, Bassi C, Thayer SP, Falconi M, Mantovani W, et al: Main-duct intraductal papillary mucinous neoplasms of the pancreas: clinical predictors of malignancy and long-term survival following resection. Ann Surg 2004;239: 678-685; discussion 85-87.

28 Ferrone CR, Correa-Gallego C, Warshaw AL, Brugge WR, Forcione DG, Thayer SP, et al: Current trends in pancreatic cystic neoplasms. Arch Surg 2009;144:448-454.

29 Gaujoux S, Brennan MF, Gonen M, D’Angelica MI, DeMatteo R, Fong Y, et al: Cystic lesions of the pancreas: changes in the presentation and management of 1,424 patients at a single institution over a 15-year time period. J Am Coll Surg 2011;212:590600; discussion 600-603.

-30 Tanaka M, Chari S, Adsay V, Fernandez-del Castillo C, Falconi M, Shimizu M, et al: International consensus guidelines for management of intraductal papillary mucinous neoplasms and mucinous cystic neoplasms of the pancreas. Pancreatology 2006;6:1732 .

-31 Fernandez-del Castillo C, Targarona J, Thayer SP, Rattner DW, Brugge WR, Warshaw AL: Incidental pancreatic cysts: clinicopathologic characteristics and comparison with symptomatic patients. Arch Surg 2003;138:427-423; discussion 33-34.

-32 Crippa S, Fernandez-Del Castillo C, Salvia R, Finkelstein D, Bassi C, Dominguez I, et al: Mucin-producing neoplasms of the pancreas: an analysis of distinguishing clinical and epidemiologic characteristics. Clin Gastroenterol Hepatol 2010;8:213-219.

-33 Sohn TA, Yeo CJ, Cameron JL, Hruban RH, Fukushima N, Campbell KA, et al: Intraductal papillary mucinous neoplasms of the pancreas: an updated experience. Ann Surg 2004;239:788-797; discussion 97-99.

-34 Matsumoto T, Aramaki M, Yada K, Hirano S, Himeno Y, Shibata K, et al: Optimal management of the branch duct type intraductal papillary mucinous neoplasms of the pancreas. J Clin Gastroenterol 2003;36:261265.
35 Koito K, Namieno T, Ichimura T, Yama N, Hareyama M, Morita K, et al: Mucin-producing pancreatic tumors: comparison of MR cholangiopancreatography with endoscopic retrograde cholangiopancreatography. Radiology 1998;208:231-237.

36 Salvia R, Bassi C, Falconi M, Serini P, Crippa $\mathrm{S}$, Capelli $\mathrm{P}$, et al: Intraductal papillary mucinous tumors of the pancreas. Surgical treatment: at what point should we stop? JOP 2005;6(1 suppl):112-117.

37 Bernard P, Scoazec JY, Joubert M, Kahn X, Le Borgne J, Berger F, et al: Intraductal papillary-mucinous tumors of the pancreas: predictive criteria of malignancy according to pathological examination of 53 cases. Arch Surg 2002;137:1274-1278.

-38 Ahmad NA, Kochman ML, Lewis JD, Ginsberg GG: Can EUS alone differentiate between malignant and benign cystic lesions of the pancreas? Am J Gastroenterol 2001;96: 3295-3300.

-39 Volmar KE, Vollmer RT, Jowell PS, Nelson RC, Xie HB. Pancreatic FNA in 1000 cases: a comparison of imaging modalities. Gastrointest Endosc 2005;61:854-861.

40 Brugge WR, Lewandrowski K, Lee-Lewandrowski E, Centeno BA, Szydlo T, Regan S, et al: Diagnosis of pancreatic cystic neoplasms: a report of the cooperative pancreatic cyst study. Gastroenterology 2004;126:13301336.

41 Sarr MG, Murr M, Smyrk TC, Yeo CJ, Fernandez-del-Castillo C, Hawes RH, et al: Primary cystic neoplasms of the pancreas. Neoplastic disorders of emerging importancecurrent state-of-the-art and unanswered questions. J Gastrointest Surg 2003;7:417428.

42 Mansour JC, Schwartz L, Pandit-Taskar N, D'Angelica M, Fong Y, Larson SM, et al: The utility of F-18 fluorodeoxyglucose whole body PET imaging for determining malignancy in cystic lesions of the pancreas. J Gastrointest Surg 2006;10:1354-1360.

43 Procacci C, Biasiutti C, Carbognin G, Accordini S, Bicego E, Guarise A, et al: Characterization of cystic tumors of the pancreas: CT accuracy. J Comput Assist Tomogr 1999;23: 906-912. 